\title{
Semi-intensive and Intensive Interdisciplinary Treatments Have Similar Effects on Metabolic Syndrome and Selected Inflammatory Markers in Adolescents with Obesity
}

\author{
Luzia Jaeger Hintze ${ }^{1, *}$, Ana Claudia Pelissari Kravchychyn', Yasmin Alaby Martins Ferreira', Raquel Munhoz da Silveira Campos², \\ Alexandre D. Aguilera Dantas ${ }^{3}$, Deborah Cristina Landi Masquio ${ }^{4}$, Danielle Arisa Caranti ${ }^{2}$, David Thivel ${ }^{5}$, Ana R. Dâmaso ${ }^{1}$ \\ ${ }^{1}$ Department of Biosciences, Post Graduate Program of Nutrition, Universidade Federal de São Paulo, São Paulo; ${ }^{2}$ Department of Biosciences, Post Graduate Program \\ of Interdisciplinary Health Sciences, Universidade Federal de São Paulo, Campus Baixada Santista, Santos; ${ }^{3}$ Brazilian Center of Sports and Nutrition, São Paulo; \\ ${ }^{4}$ São Camilo University Center; São Paulo, Brazil, ${ }^{5}$ Clermont Auvergne University, EA 3533, Laboratory of the Metabolic Adaptations to Exercise under Physiological \\ and Pathological Conditions (AME2P), Clermont-Ferrand, France
}

Background: The purpose of the present study was to compare the impact of semi-intensive and intensive interdisciplinary weight-loss therapies on the treatment of metabolic syndrome (MS) and selected inflammatory markers in adolescents with obesity.

Methods: The study included 166 adolescents enrolled in two groups for 22 weeks: the intensive group (inperson aerobic and resistance exercise three times a week, and psychological and nutritional counselling once a week), or the semi-intensive group (six in-person exercise orientation meetings and six in-person psychological support sessions with an online nutritional and exercise program). Anthropometric parameters, blood pressure, fasting glucose, insulin, lipid profile, triglycerides (TG), leptin and adiponectin were assessed before and after the interventions.

Results: Body weight and waist circumference decreased in both groups $(P<0.001)$ and large effect sizes $\left(\eta^{2}=\right.$ 0.586 and $\eta^{2}=0.465$, respectively) were noted. Significant time and group interactions were found $(P=0.001)$ with medium effect sizes ( $\eta^{2}=0.095$ and $\eta^{2}=0.105$, respectively). The prevalence of MS decreased from $27.9 \%$ to $13.1 \%(P=0.012)$ and $29.4 \%$ to $5.9 \%(P=0.004)$ in the semi-intensive and intensive groups, respectively. All MS risk factors decreased significantly over time $(P<0.001)$ and a significant time*group interaction was observed $(P<0.05)$, except for fasting TG $(P=0.832)$ and glucose $\left(P=0.128, \eta^{2}=0.021\right)$.

Conclusion: The results suggested that both approaches promoted significant clinical improvement in the condition of adolescents with MS and reduced associated risk factors. Studies that consider the cost effectiveness of both treatments are still needed to determine whether semi-intensive care, with its lower financial costs, may be a suitable option to treat obesity and MS in adolescents with obesity.

Key words: Metabolic syndrome, Adolescents, Leptin, Adiponectin, Patient care team, Weight loss, Obesity management
Received April 23, 2021

Reviewed September 10, 2021

Accepted October 18, 2021

*Corresponding author

Luzia Jaeger Hintze

https://orcid.org/0000-0002-2857-7034

Department of Biosciences, Post Graduate Program of Nutrition, Escola Paulista de Medicina, Universidade Federal de Sao Paulo, 862, Botucatu Street 2nd floor, Vila Clementino, São Paulo 04023-062, Brazil Tel: +55-11-55764765

E-mail: lujhintze@hotmail.com

\section{INTRODUCTION}

The increasing worldwide prevalence of obesity and its negative consequences have been exhaustively reported in the literature. Im- portantly, recent data indicates an alarming global increase of about $76 \%$ in the prevalence of obesity among adolescents over the last four decades. ${ }^{1}$ In Brazil, the prevalence of obesity in children and adolescents is approximately $14 \% .^{2}$ Obesity is associated with in-

Copyright @ 2021 Korean Society for the Study of Obesity

(a) This is an Open Access article distributed under the terms of the Creative Commons Attribution Non-Commercial License (https://creativecommons.org/licenses/by-nc/4.o/) which permits unrestricted non-commercial use, distribution, and reproduction in any medium, provided the original work is properly cited. 
creased blood pressure (BP), type 2 diabetes, non-alcoholic liver steatosis, osteoarthritis, inflammatory states or metabolic syndrome (MS), and cardiometabolic diseases, among other conditions.,

The MS is commonly defined as the co-occurrence of three or more cardiometabolic risk factors, such as increased waist circumference (WC), BP, fasting blood glucose, and/or serum triglyceride (TG) concentrations, and decreased high-density lipoprotein cholesterol (HDL-C). Studies conducted in Brazil have shown that approximately $30 \%$ of adolescents with obesity present with MS., which has particular importance given MS has been shown to be associated with lower responses to weight-loss treatments and reduced improvement in lipid and inflammatory profiles. ${ }^{7}$ Individuals with obesity tend to present with elevated serum leptin concentrations (hyperleptinemic state) and reduced serum adiponectin, which negatively impact the regulation of energy balance. ${ }^{8,9}$

Therefore, MS interventions should not only focus on weight loss, but also on strategies targeting the risk factors associated with MS. ${ }^{10}$ Interdisciplinary treatment involving professionals such as kinesiologists, psychologists, dietitians, physicians and physiotherapists has been shown to provide good results in reducing cardiometabolic risks in adults and adolescents with obesity. ${ }^{4,7}$ However, implementing large-scale interventions involving multiple professionals is costly for public health systems. There has, therefore, been an ongoing search for alternative low-cost and effective strategies to treat obesity and its health-related consequences: proposed approached have included the use of electronic technologies, including the internet and web-based programs, as adjuvants in health education. ${ }^{11}$ Previous studies have indicated that these types of interventions can help to reduce body weight and improve several health-related issues associated with obesity, such as type II diabetes, ${ }^{12,13}$ hypertension, increased TG, and reduced HDL-C. ${ }^{14,15}$

Although these results appear very promising, further studies are required to confirm the earlier findings. It has previously been observed that more intensive therapies produce greater impact on body-weight variables; however, the extent of the impact of intensive therapies on MS prevalence and inflammatory markers compared to semi-intensive therapies remains uncertain in adolescents. Accordingly, the objective of the present study was to compare the effects of intensive and semi-intensive interdisciplinary approaches on anthropometric parameters, body composition, MS and inflam- matory markers. We hypothesized that the intensive therapy would have greater impact on anthropometric and body composition variables, but that both approaches would produce clinically significant reductions in MS risk factors and inflammatory markers.

\section{METHODS}

\section{Research design}

The present study involved a total of 166 adolescents with obesity, aged between 14 and 19 years (average, $17.1 \pm 1.7$ years). The main inclusion criteria were as follows: $\geq$ stage 5 on the Tanner scale; and a body mass index (BMI) $\geq 95$ th percentile. Participants who reported the use of birth-control pills, cortisone, anti-epileptic drugs, and a history of renal disease, alcohol intake, smoking, and secondary obesity due endocrine disorders were not included in the study. Adolescents who reported orthopedic limitations and/ or other alterations indicated in the electrocardiogram that did not allow the participants to join in the physical exercise sessions were also not included. The study was conducted according to the principles of the Declaration of Helsinki and was approved by the Ethics Committee on Research at the Sao Paulo Federal UniversityUNIFESP (\#0135/04 and \#0052/16). Adolescents and their legal guardians received comprehensive information about the trials before signing assent or consent forms.

The present study compared two different and independent clinical interventions (intensive and semi-intensive) that were implemented in our health department over different time periods. Participants were assigned to the groups (intensive weight-loss therapy or semi-intensive weight-loss therapy) by convenience sampling (Fig. 1).

\section{Interdisciplinary therapy weight-loss models}

After a screening session, the adolescents either followed a 22-week intensive or semi-intensive interdisciplinary weight-loss intervention. All face-to-face interventions and assessments took place at the Federal University of Sao Paulo. In-person nutritional and psychological support meetings took place every week (60 minutes) in the intensive group, and in-person exercise sessions, which included aerobic and resistance training, took place three times per week. In the semi-intensive group, there were six in-person exercise orien- 


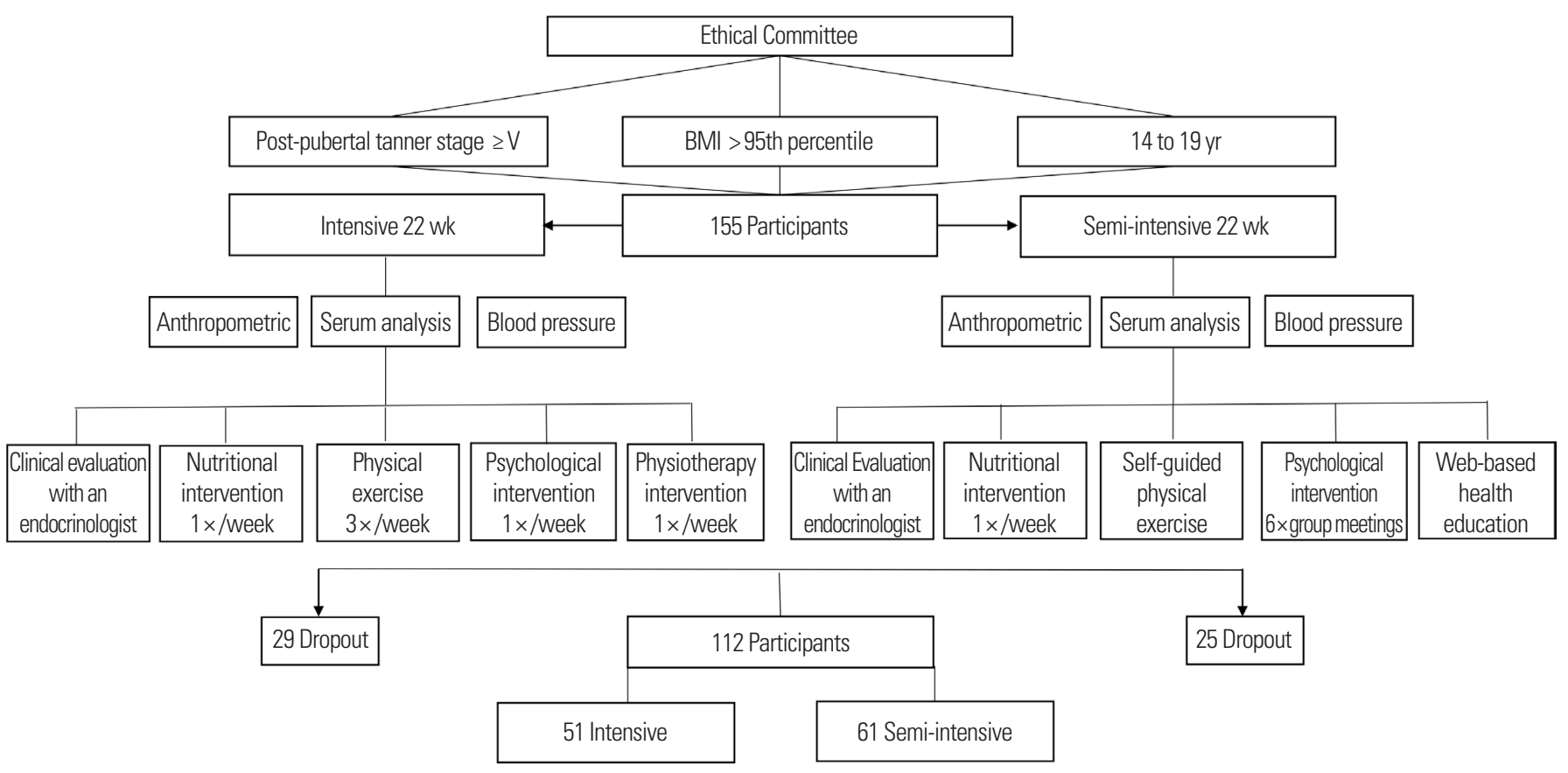

Figure 1. Study design. BMI, body mass index.

tation sessions, with the participants following on-line classes in the other sessions. The semi-intensive group received health education once per week and was remotely monitored online. Only six inperson psychological support meetings were offered to this group. An in-person clinical examination of participants from both groups took place at baseline and information on family history was collected. In-person interventions to address any health problems that adolescents from either group developed over the course of the intervention.

\section{Nutritional therapy}

The nutritional intervention was conducted by a registered dietician. For both groups daily energy intake was individually planned according to the recommendations for adolescents with low levels of physical activity, considering age and sex. The distribution of macronutrients was as follows: fat (25\%-35\%), carbohydrate (45\%$65 \%)$, and protein (10\%-30\%). Participants in the intensive group had weekly face-to-face group lessons regarding healthy eating behaviors, whereas the participants from the semi-intensive group had online access to weekly recorded lessons (access was available only during the duration of the program). The lesson topics were similar for both groups. ${ }^{16}$

\section{Exercise protocols}

In the intensive group, the in-person physical exercise program was performed three times weekly and included 30 minutes of aerobic training plus 30 minutes of resistance training per session. ${ }^{17}$ The aerobic training consisted of walking/running on a motor-driven treadmill (Life Fitness, Franklin Park, IL, USA) or using an exercise bike at a cardiac frequency intensity between ventilatory thresholds 1 and $2( \pm 4 \mathrm{bpm})$ according to the results of an initial oxygen uptake test for aerobic exercises. Resistance training was prescribed individually as described previously. ${ }^{3}$ In this group, a certified physical therapist conducted weekly sessions that were focused on subjects such as global postural re-education and other topics related to better exercise performance and safety.

In the semi-intensive group, the adolescents used a self-guided program to select the physical activities that they wanted to undertake. ${ }^{11}$ In the in-person orientation sessions that members of this group received, they were advised to undertake their chosen exercise for 60 minutes three times a week as part of the weight-loss program. In the various online program health themes, the volunteers had access to videos about the correct way to exercise, including the frequency, intensity and volume, to help them in their choices. 


\section{Psychological therapy}

Collective psychological sessions were organized once a week in the intensive therapy group and there were six in-person group meetings for the semi-intensive group over the course of the program. The topics discussed by the psychologist in both therapy models were as follows: depression, eating disorders, anxiety, low self-esteem, and body-image disorders.

\section{Anthropometric, body composition and BP}

\section{measurements}

Body weight, height, and WC were measured using standard procedures. Systolic blood pressure (SBP) and diastolic blood pressure (DBP) were measured in the right arm using a mercury-gravity manometer with appropriate cuff size. Body composition in the intensive group was measured using air-displacement plethysmography as performed in a BOD POD body composition system (version 1.69; Life Measurement Instruments, Concord, CA, USA), while a Bioimpedance 310E meter (Biodynamics Corp., Shoreline, WA, USA) was used for the semi-intensive group. Previous research has demonstrated good agreement between both techniques. ${ }^{18}$

\section{Serum analyses}

Blood samples were collected after a 12-hour overnight fast at baseline and at the end of each intervention. The serum was separated into serum and plasma and concentrations of glucose, TG and HDL-C determined by enzymatic colorimetric methods (Celm, Barueri, Brazil). The adipokines leptin and adiponectin were measured with enzyme-linked immunosorbent assay kits (R\&D Systems, Minneapolis, MN, USA). Hyperleptinemia was diagnosed using 20 and $24 \mu \mathrm{g} / \mathrm{mL}$ as the cut-off points for boys and girls, respectively. In all cases, MS was diagnosed according to the International Diabetes Federation criteria. ${ }^{19}$

\section{Statistical analyses}

All data were analyzed using SPSS version 15.0 (SPSS Inc., Chicago, IL, USA). The results were presented as the mean \pm standard deviation, and as counts and percentages if the data were categorical. Differences in the prevalence of MS, MS risk factors and hyperleptinemia were calculated using McNemar's test. Two-way repeated measures analysis of variances were performed to assess changes in body weight, body composition, MS risk factors, and inflammatory indicators at baseline and post weight loss within each group and between groups (time $\times$ group interaction). Effects were considered significant at $P<0.05$. Moreover, in order to assess the magnitude of potential observed differences, the effect size was computed (eta squared, $\eta^{2}$ ). The values of $0.010,0.059$, and 0.138 were considered cut-off values for small, medium, and large effect sizes.

\section{RESULTS}

During the intervention period, 29 participants (36.2\%) from the intensive group and 25 participants (29\%) from the semi-intensive group dropped out of the study. The main reasons given for dropping out were job opportunities and school schedules, followed by financial and family problems. No significant differences were noted $(P=0.516)$ between the groups with respect to dropout percentages. In the intensive group, we only included participants who attended at least $75 \%$ of the face-to-face sessions. A total of $112 \mathrm{ad}-$ olescents from both groups completed the intervention and were included in the analyses (intensive: $\mathrm{n}=51$; semi-intensive: $\mathrm{n}=61$ ). Table 1 shows the demographic information for both groups at baseline. Neither age nor BMI differed between the groups at baseline ( $P=0.723$ and $P=0.921$, respectively), showing that both groups were similar at the onset of the study.

Changes in anthropometric and body composition measurement results in those who completed the intensive and semi-intensive interventions are shown in Table 2. Weight loss after the interventions was $-5.05 \%(4.24, P<0.001)$ and $-9.43 \%(7.14, P<0.001)$ in the semi-intensive and intensive groups, respectively. We found significant time $\times$ group effects $(P<0.001)$ for all anthropometric and body composition measurements (except for height), with the in-

Table 1. Baseline demographic characteristics of the study population

\begin{tabular}{lccc}
\hline Variable & Semi-intensive & Intensive & $P$ \\
\hline Age $(y r)$ & $17.15(1.75)$ & $17.03(1.67)$ & 0.723 \\
Baseline BMl & $38.2(4.54)$ & $38.3(5.34)$ & 0.921 \\
Drop-out & $25(29)$ & $29(36.2)$ & 0.516 \\
No. of completers & 61 & 51 & 0.064 \\
Female & $31(51)$ & $34(67)$ & \\
Male & $30(49)$ & $17(33)$ & \\
\hline
\end{tabular}

Values are presented as number (\%).

$\mathrm{BMI}$, body mass index. 
Table 2. Anthropometric measurement results and body composition at baseline and post intervention in the semi-intensive and intensive groups

\begin{tabular}{|c|c|c|c|c|c|c|c|c|c|c|}
\hline \multirow{2}{*}{ Variable } & \multicolumn{3}{|c|}{ Semi-intensive $(n=61)$} & \multicolumn{3}{|c|}{ Intensive $(n=51)$} & \multirow{2}{*}{$\begin{array}{c}\text { Change within } \\
\text { groups }(P)\end{array}$} & \multirow{2}{*}{$\begin{array}{c}\text { Effect size } \\
\eta^{2}\end{array}$} & \multirow{2}{*}{$\begin{array}{l}\text { Change between } \\
\text { groups }(P)\end{array}$} & \multirow{2}{*}{$\begin{array}{c}\text { Effect size } \\
\eta^{2}\end{array}$} \\
\hline & Baseline & Postintervention & $\%$ Change & Baseline & Postintervention & $\%$ Change & & & & \\
\hline BW (kg) & $110.99 \pm 15.22$ & $105.28 \pm 14.41$ & -5.15 & $107.09 \pm 16.31$ & $97.11 \pm 17.65$ & -9.30 & $<0.001^{*}$ & 0.586 & $0.001^{*}$ & 0.095 \\
\hline BW z score & $0.11 \pm 0.96$ & $0.23 \pm 0.87$ & & $-0.13 \pm 1.03$ & $-0.27 \pm 1.07$ & & $0.821^{*}$ & 0.000 & $0.002^{*}$ & 0.085 \\
\hline Height (m) & $1.71 \pm 0.09$ & $1.71 \pm 0.09$ & 0.32 & $1.67 \pm 0.08$ & $1.68 \pm 0.08$ & 0.28 & $<0.001^{*}$ & 0.016 & 0.863 & 0.000 \\
\hline Height z score & $0.17 \pm 1.02$ & $0.17 \pm 1.01$ & & $-0.19 \pm 0.94$ & $-0.20 \pm 0.96$ & & $0.992^{*}$ & 0.000 & 0.885 & 0.000 \\
\hline $\mathrm{BMI}\left(\mathrm{kg} / \mathrm{m}^{2}\right)$ & $38.21 \pm 4.54$ & $36.05 \pm 4.56$ & -5.70 & $38.30 \pm 5.34$ & $34.54 \pm 5.80$ & -9.80 & $<0.001^{*}$ & 0.622 & $<0.001^{*}$ & 0.107 \\
\hline BMl z-score & $-0.01 \pm 0.92$ & $0.13 \pm 0.87$ & & $0.01 \pm 1.09$ & $-0.15 \pm 1.11$ & & $0.802^{*}$ & 0.001 & $0.001^{*}$ & 0.103 \\
\hline Fat mass (kg) & $41.99 \pm 8.44$ & $37.89 \pm 7.40$ & -9.03 & $51.33 \pm 12.61$ & $37.96 \pm 14.06$ & -27.16 & $<0.001^{*}$ & 0.691 & $<0.001^{*}$ & 0.387 \\
\hline Lean body mass (kg) & $68.65 \pm 10.71$ & $67.04 \pm 10.38$ & -2.26 & $55.55 \pm 7.41$ & $59.42 \pm 9.33$ & 6.48 & $0.002^{*}$ & 0.083 & $<0.001^{*}$ & 0.348 \\
\hline
\end{tabular}

Values are presented as mean \pm standard deviation.

${ }^{*}$ Significant differences; effect sizes $\eta^{2}=0.010,0.059$, and 0.138 were cut-off points for small, medium, and large effect sizes, respectively.

$\mathrm{BW}$, body weight; BMl, body mass index.

Table 3. MS risk factors and inflammatory biomarkers at baseline and post intervention in the semi-intensive and intensive groups

\begin{tabular}{|c|c|c|c|c|c|c|c|c|c|c|}
\hline \multirow{2}{*}{ Variable } & \multicolumn{3}{|c|}{ Semi-intensive $(n=61)$} & \multicolumn{3}{|c|}{ Intensive $(n=51)$} & \multirow{2}{*}{$\begin{array}{l}\text { Change within } \\
\text { group }(P)\end{array}$} & \multirow{2}{*}{$\begin{array}{l}\text { Effect } \\
\text { size } \eta^{2}\end{array}$} & \multirow{2}{*}{$\begin{array}{c}\text { Change between } \\
\text { group }(P)\end{array}$} & \multirow{2}{*}{$\begin{array}{l}\text { Effect } \\
\text { size } \eta^{2}\end{array}$} \\
\hline & Baseline & Post-intervention & $\%$ Change & Baseline & Post-intervention & $\%$ Change & & & & \\
\hline $\mathrm{SBP}(\mathrm{mmHg})$ & $118.11 \pm 9.75$ & $119.11 \pm 8.50$ & 1.31 & $125.85 \pm 18.39$ & $114.79 \pm 7.87$ & -6.59 & $<0.001^{*}$ & 0.622 & $<0.001^{*}$ & 0.107 \\
\hline $\mathrm{DBP}(\mathrm{mmHg})$ & $76.64 \pm 6.37$ & $76.88 \pm 6.71$ & 0.70 & $79.15 \pm 8.75$ & $74.15 \pm 5.75$ & -6.31 & $0.006^{*}$ & 0.070 & $0.002^{*}$ & 0.083 \\
\hline WC (cm) & $109.86 \pm 10.09$ & $104.97 \pm 10.80$ & -4.41 & $105.72 \pm 11.26$ & $95.13 \pm 12.06$ & -11.00 & $<0.001^{*}$ & 0.465 & $0.001^{*}$ & 0.105 \\
\hline Glucose (mg/dL) & $89.62 \pm 7.47$ & $88.44 \pm 7.28$ & -0.78 & $90.57 \pm 7.77$ & $86.76 \pm 7.55$ & -5.30 & $0.004^{*}$ & 0.072 & 0.128 & 0.021 \\
\hline $\mathrm{HDL}-\mathrm{C}$ (mg/dL) & $40.51 \pm 8.33$ & $43.23 \pm 8.28$ & 8.08 & $43.55 \pm 8.45$ & $44.06 \pm 9.15$ & 3.90 & $0.003^{*}$ & 0.078 & $0.039^{*}$ & 0.038 \\
\hline $\mathrm{TG}(\mathrm{mg} / \mathrm{dL})$ & $127.25 \pm 58.41$ & $111.25 \pm 57.87$ & -7.84 & $106.67 \pm 75.70$ & $88.35 \pm 45.70$ & -9.76 & $0.002^{*}$ & 0.083 & $0.832^{*}$ & 0.000 \\
\hline Adiponectin (mg/dL) & $2.58 \pm 1.91$ & $3.34 \pm 2.93$ & 29.45 & $7.20 \pm 3.33$ & $9.39 \pm 4.76$ & 30.42 & $<0.001^{*}$ & 0.215 & $0.010^{*}$ & 0.061 \\
\hline Leptin (mg/dL) & $64.68 \pm 42.40$ & $48.35 \pm 39.04$ & -23.52 & $44.20 \pm 30.34$ & $29.83 \pm 24.70$ & -25.27 & $<0.001^{*}$ & 0.236 & 0.709 & 0.001 \\
\hline Number of MS risk factors & $2.07 \pm 0.87$ & $1.70 \pm 0.76$ & -17.90 & $2.12 \pm 0.95$ & $1.28 \pm 0.78$ & -39.60 & $<0.001^{*}$ & 0.317 & $0.005^{*}$ & 0.069 \\
\hline Adiponectin/leptin & $0.04(0.07)$ & $0.08(0.01)$ & 66.00 & $0.27(0.36)$ & $0.38(0.54)$ & 40.00 & & & 0.714 & \\
\hline
\end{tabular}

Values are presented as mean \pm standard deviation or median (interquartile range)

*Significant differences; effect sizes $\eta^{2}=0.010,0.059$, and 0.138 were cut-off points for small, medium, and large effect sizes, respectively.

MS, metabolic syndrome; SPB, systolic blood pressure; DBP, diastolic blood pressure; WC, waist circumference; HDL-C, high-density lipoprotein cholesterol; TG, triacylglycerol

tensive intervention having a greater impact with respect to all these variables. Large effect sizes for time $\times$ group effects were evident for all body composition variables except for body weight $\left(\eta^{2}=0.095\right)$. Comparisons of the $\% \Delta$ also indicated significant differences $(P<$ $0.001)$ in all body composition and anthropometric variables except for height $(P=0.863)$.

The risk factors for MS and associated inflammatory parameters are described in Table 3. We found the participants' WC, glucose, TG and leptin decreased significantly in both groups $(P<0.001)$, whereas SBP and DBP decreased only in the intensive group $(P<$ 0.001 ). In addition, DBP, TG and glucose presented medium effect sizes $\left(\eta^{2}=0.070, \eta^{2}=0.083\right.$, and $\eta^{2}=0.072$, respectively), whereas SBP, WC and leptin presented large effect sizes $\left(\eta^{2}=0.622, \eta^{2}=\right.$ 0.465 , and $\eta^{2}=0.236$, respectively). Adiponectin and HDL-C sig- nificantly increased $(P<0.001$ and $P=0.003$, respectively), with a large effect size for adiponectin $\left(\eta^{2}=0.215\right)$ and a medium effect size for HDL-C $\left(\eta^{2}=0.078\right)$. Significant time $\times$ group effects were found for SBP, DBP, WC, HDL-C and adiponectin. However, medium effect sizes were found for DBP and adiponectin $\left(\eta^{2}=0.083\right.$ and $\eta^{2}=0.061$, respectively) and a small effect size was noted for HDL-C $\left(\eta^{2}=0.038\right)$. Glucose, leptin and TG did not have significant time $\times$ group effects, and also had small effect sizes, suggesting that both interventions promoted similar impact in these variables. The number of MS risk factors decreased over time $(P<0.001$, $\left.\eta^{2}=0.317\right)$ and there was a time $\times$ group effect in these variables $(P=0.005)$; however, a medium effect size $\left(\eta^{2}=0.069\right)$ was observed. The $\% \Delta$ analysis also indicated significant differences between groups in SBP and DBP $(P<0.001$ and $P=0.003$, respec- 
A

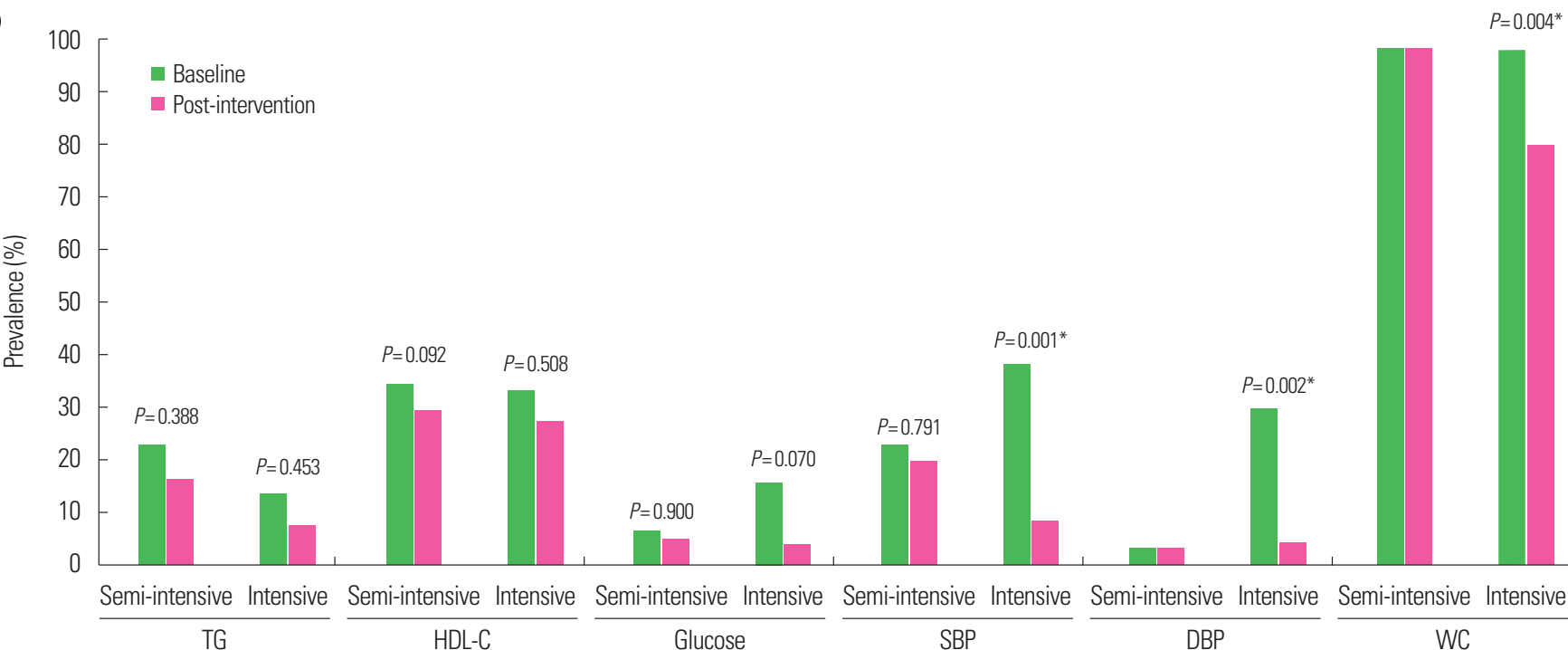

B

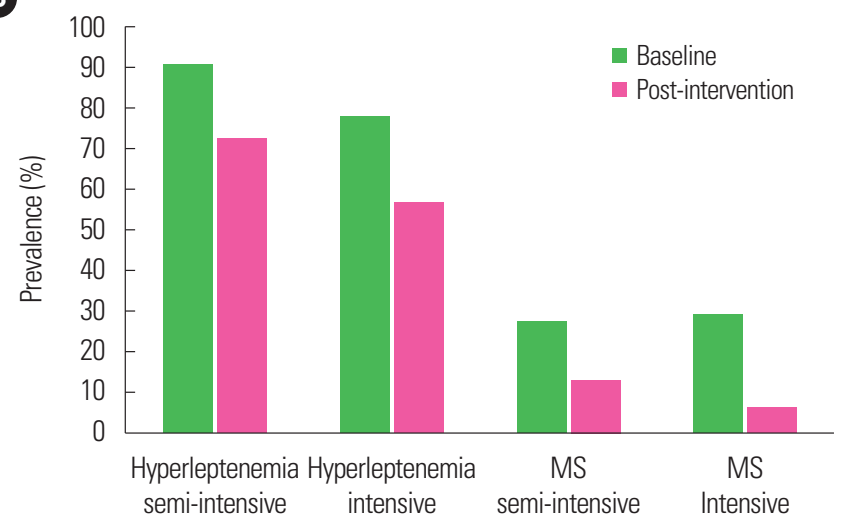

tively) and HDL-C $(P=0.023)$. No significant differences in the $\% \Delta$ were found in the analysis of glucose $(P=0.097), \mathrm{TG}(P=0.900)$, adiponectin $(P=0.951)$ or leptin $(P=0.312)$.

Fig. 2 shows the prevalence of each MS risk factor (a), and the prevalence of MS and prevalence of hyperleptinemia (b) in both groups. The SBP, DBP, and WC decreased significantly only in the intensive group ( $P=0.001, P=0.002$ and $P=0.004$, respectively). Overall, MS prevalence significantly decreased from $28.6 \%(\mathrm{n}=32)$ to $9.8 \%(\mathrm{n}=11, P=0.001)$. In the semi-intensive group, the prevalence decreased from $27.9 \%(\mathrm{n}=17)$ to $13.1 \%(\mathrm{n}=8, P=0.012)$; whereas in the intensive group it decreased from $29.4 \%(n=15)$ to $5.9 \%(\mathrm{n}=3, P=0.004)$. Hyperleptinemia also decreased from $90 \%$ $(\mathrm{n}=55)$ to $73 \%(\mathrm{n}=45, P=0.007)$ in the semi-intensive group, and from $76 \%(\mathrm{n}=56)$ to $57 \%(\mathrm{n}=30, P=0.002)$ in the intensive group.
Figure 2. Metabolic syndrome (MS) factors (A) and prevalence of MS-associated hyperleptinemia (B) in adolescents with obesity. ${ }^{*}$ Significance $P<0.05$. TG, triacylglycerol; HDL-C, high-density lipoprotein cholesterol; SPB, systolic blood pressure; $\mathrm{DBP}$, diastolic blood pressure; $\mathrm{WC}$, waist circumference.

\section{DISCUSSION}

Only a handful of studies have evaluated the impact of webbased interdisciplinary approaches on cardiometabolic risk ${ }^{12,14,15,18}$ and we are unaware of any study that compared the impact of semiintensive and intensive clinical interdisciplinary therapies on both MS risk factors and pro-anti-inflammatory biomarkers (leptin and adiponectin) in adolescents with obesity. The findings of this study only partially supported our hypothesis that both therapies would produce clinically significant reductions in MS factors and inflammatory markers, as $\mathrm{BP}$ and $\mathrm{WC}$ were only reduced in the intensive intervention group. To the best of our knowledge, the present study is the first to indicate that both intensive and semi-intensive interdisciplinary therapies have similar effects on some inflammatory markers and MS risk factors in adolescents with obesity.

Our results also demonstrated that both interventions promoted 
significant weight loss in our participants. Although the impact was greater in the intensive program, there was also significant weight loss in the semi-intensive group. Interestingly, the average weight loss of approximately $5 \%$ in our semi-intensive group was similar to that reported by some web-based interventions studies, ${ }^{15,19}$ but higher than in other studies. ${ }^{20,21}$ Even though percent weight lost was smaller in the semi-intensive group compared with the intensive group, it is likely that it was sufficient to promote health-related benefits. A relatively modest weight loss of $5 \%$ has previously been reported to reduce or even eliminate cardiometabolic risks and chronic degenerative diseases such as hypertension and hyperlipidemia, as well as being beneficial for control of hyperglycemia. ${ }^{15,22}$

Consistent with our results, previous studies involving adolescents with obesity also showed significant improvements in fat mass (FM). Changes in FM in our semi-intensive group (-9\% FM reduction) were smaller than the results reported for other intensive therapies, ${ }^{23,24}$ but greater than those of other web-based intervention studies. ${ }^{12,14}$ Reducing FM has been found to be associated with improvements in cardiometabolic risk, ${ }^{7}$ and there is evidence indicating that this improvement can occur independently from weight and fat losses as long as participants change lifestyle behaviors. ${ }^{24}$ Web-based therapies may, therefore, be helpful tools to promote and improve cardiometabolic risk. Indeed, previous studies have indicated the positive impact of web-based therapies, which resulted in reduced fat intake, increased physical activity, ${ }^{25}$ and reduced weekly screen time. ${ }^{21}$

The analysis of inflammatory markers indicated significant changes in serum adiponectin and leptin concentrations and in the adiponectin/leptin ratio, with adiponectin significantly increasing and leptin significantly decreasing over time. The prevalence of hyperleptinemia also decreased significantly in both groups. These changes favor improved energy metabolism and are associated with a better weight loss response. ${ }^{4,8}$ The results of previous research conducted in our laboratory indicated that control of hyperleptinemia and increased adiponectin concentrations are associated with better vascular protection ${ }^{4}$ and reductions in eating disorder symptoms. ${ }^{26}$ Given the role of both peptides in energy metabolism, better control of their concentrations in serum might favor long-term weight loss maintenance.

Previous studies have suggested that the adiponectin/leptin ratio is an important clinical marker of adipose tissue dysfunction. ${ }^{27} \mathrm{~A}$ reduction of this ratio is an expected a consequence of weight loss. Interestingly, we noted the adiponectin/leptin ratio decreased in the semi-intensive group. In fact, previous studies from our group indicated significant increases in the adiponectin/leptin ratio after intensive treatment. ${ }^{47,27}$ To the best of our knowledge, no other studies have investigated adiponectin/leptin ratio after semi-intensive weight loss programs and our results need to be confirmed in future studies.

Another interesting finding from the present study is the significant reduction in the prevalence of MS in both intervention groups. The number of MS risk factors also decreased. Previous studies demonstrated the positive impact of an interdisciplinary weight-loss treatment on MS, ${ }^{7}$ even after only a three-week weight reduction program; ${ }^{28}$ however, few studies have investigated the impact of web-based approaches on this variable. Block et al. ${ }^{12}$ demonstrated a significant reduction in MS prevalence in their study with adults. Among the participants who had MS at baseline, $46.5 \%$ of those in a web-based group no longer had MS after a six-month intervention. Another study ${ }^{28}$ reported significant reductions in BMI, FM, and body weight among other improvements in boys and girls with obesity, with and without MS, after a three-week multidisciplinary body-weight reduction program. These results indicate that even though the treatments or interventions might not produce complete remission of MS, they may still provide many other clinical benefits.

Unlike previous studies, in our study there were improvements in BP only in the intensive group. Significant impacts of web-based and mobile-phone-based therapies on BP have been reported. ${ }^{14}$ However, most of these studies compared results between a nontreatment control group and a web-based intervention group..$^{14,18}$ Other studies included only personalized phone messages as complement to the participants' regular care, ${ }^{29,30}$ which might explain the differences between our findings and those reported in other studies. The differences in weight loss between the groups might also explain the significant BP reduction only in the intensive group, given the association between high $\mathrm{BP}$ and body weight.

Conversely, our study demonstrated significant effects on TG and HDL-C. The effects of intensive therapies on these variables is well recognized. ${ }^{3,4,7}$ Web-based interventions have also been found 
to promote significant changes in these variables. A reduction of HDL-C in the first 12 weeks of intervention was reported in adults; ${ }^{14,15,18}$ whereas other authors showed decreases in TG after 12 weeks of web-based weight-loss treatment. ${ }^{12,15}$ Interestingly, in our study we did not find a significant time $\times$ group effect for TG and only a small effect size was found for HDL-C, which indicated that both interventions may influence these two MS risk factors to a similar degree.

The efficacy of web-based programs to control hyperglycemia and other glycemic markers in adults has been previously reported. ${ }^{12,13}$ Similar to previous studies, we found a significant improvement in fasting glucose concentrations in our participants and no time $\times$ group interactions were noted. Indeed, previous studies have reported that diabetes risk might be reduced by even modest weight loss. ${ }^{12} \mathrm{Kim}$ and $\mathrm{Kim}^{13}$ observed that patients with type II diabetes that received personalized and continuous recommendations addressing diet, exercise, and medication adjustment, as well as frequent self-monitoring of blood glucose concentrations, had better glycemic control after a 12-month intervention.

The present study had some limitations. Firstly, we compared two different clinical interventions that were implemented in our health department and no randomization was applied. However, our statistical analyses demonstrated no differences in age and BMI between the two groups at baseline, which allowed the analyses. Secondly, we included no measures of adherence in the study. However, we observed similar drop-out rates in the two groups and we included only participants from the intensive group who attended at least $75 \%$ of the face-to-face sessions. Finally, we did not use the same instruments to measure body composition in our participants. However, previous studies indicated good agreement between the two methods included in the study. ${ }^{18}$ Our study is also strengthened by its novelty, and by the use of gold-standard techniques in the outcome measures. Other aspects of our methods, such as the well-structured intensive intervention and the experience of our team in dealing with individuals with obesity, may also strengthen the internal validity of our results.

In conclusion, our results suggest that both interventions might be suitable options for adolescents seeking treatment for health-related issues associated with obesity, including increased inflammatory biomarkers and the presence of one or more MS risk factors.
The intensive intervention seemed to promote a greater impact in terms of anthropometric and body composition variables, inflammatory markers and MS. The results of the semi-intensive treatment were nonetheless significant, except with respect to $\mathrm{BP}$ and WC, and given web-based interventions are less costly, should be considered a valid treatment option. Further studies comparing the cost effectiveness of the two interventions are warranted before implementing web-based/semi-intensive programs on a population basis.

\section{CONFLICTS OF INTEREST}

The authors declare no conflict of interest.

\section{ACKNOWLEDGMENTS}

This work was supported by a National Council for Scientific and Technological Development (CNPq) grant \#409943/2016-9 and \#301322/2017-1. LJH received a scholarship (CNPq - Postdoc fellow \#58589/2018-1).

\section{AUTHOR CONTRIBUTIONS}

Study concept and design: LJH, DT, and ARD; acquisition of data: ACPK, YAMF, RMSC, ADAD, DCLM, and DAC; analysis and interpretation of data: $\mathrm{LJH}, \mathrm{RMSC}$, DT, and ARD; drafting of the manuscript: $\mathrm{LJH}, \mathrm{ACPK}$, YAMF, RMSC, ADAD, DCLM, and DAC; critical revision of the manuscript: DT and ARD; statistical analysis: LJH and RMSC; obtained funding: LJH, DT, and ARD; administrative, technical, or material support: ACPK, YAMF, RMSC, ADAD, DCLM, and DAC; and study supervision: DT and ARD.

\section{REFERENCES}

1. NCD Risk Factor Collaboration (NCD-RisC). Worldwide trends in body-mass index, underweight, overweight, and obesity from 1975 to 2016: a pooled analysis of 2416 populationbased measurement studies in 128.9 million children, adolescents, and adults. Lancet 2017;390:2627-42.

2. Aiello AM, Marques de Mello L, Souza Nunes M, Soares da 
Silva A, Nunes A. Prevalence of obesity in children and adolescents in Brazil: a meta-analysis of cross-sectional studies. Curr Pediatr Rev 2015;11:36-42.

3. Dâmaso AR, de Piano A, Campos RM, Corgosinho FC, Siegfried W, Caranti DA, et al. Multidisciplinary approach to the treatment of obese adolescents: effects on cardiovascular risk factors, inflammatory profile, and neuroendocrine regulation of energy balance. Int J Endocrinol 2013;2013:541032.

4. Sanches PL, de Mello MT, Elias N, Fonseca FA, Campos RM, Carnier J, et al. Hyperleptinemia: implications on the inflammatory state and vascular protection in obese adolescents submitted to an interdisciplinary therapy. Inflammation 2014;37: 35-43.

5. Wickham EP, Stern M, Evans RK, Bryan DL, Moskowitz WB, Clore JN, et al. Prevalence of the metabolic syndrome among obese adolescents enrolled in a multidisciplinary weight management program: clinical correlates and response to treatment. Metab Syndr Relat Disord 2009;7:179-86.

6. Caranti DA, Lazzer S, Dâmaso AR, Agosti F, Zennaro R, de Mello MT, et al. Prevalence and risk factors of metabolic syndrome in Brazilian and Italian obese adolescents: a comparison study. Int J Clin Pract 2008;62:1526-32.

7. Masquio DC, de Piano A, Campos RM, Sanches PL, Carnier J, Corgosinho FC, et al. The role of multicomponent therapy in the metabolic syndrome, inflammation and cardiovascular risk in obese adolescents. Br J Nutr 2015;113:1920-30.

8. Dâmaso AR, de Piano A, Sanches PL, Corgosinho F, Tock L, Oyama LM, et al. Hyperleptinemia in obese adolescents deregulates neuropeptides during weight loss. Peptides 2011;32: 1384-91.

9. Corgosinho FC, de Piano A, Sanches PL, Campos RM, Silva PL, Carnier J, et al. The role of PAI-1 and adiponectin on the inflammatory state and energy balance in obese adolescents with metabolic syndrome. Inflammation 2012;35:944-51.

10. Sperling LS, Mechanick JI, Neeland IJ, Herrick CJ, Després JP, Ndumele CE, et al. The cardiometabolic health alliance: working toward a new care model for the metabolic syndrome. J Am Coll Cardiol 2015;66:1050-67.

11. Ferreira YA, Kravchychyn AC, Vicente SC, Campos RM, Tock L, Oyama LM, et al. An interdisciplinary weight loss program improves body composition and metabolic profile in adolescents with obesity: associations with the dietary inflammatory index. Front Nutr 2019;6:77.

12. Block G, Azar KM, Romanelli RJ, Block TJ, Hopkins D, Carpenter HA, et al. Diabetes prevention and weight loss with a fully automated behavioral intervention by email, web, and mobile phone: a randomized controlled trial among persons with prediabetes. J Med Internet Res 2015;17:e240.

13. Kim SI, Kim HS. Effectiveness of mobile and internet intervention in patients with obese type 2 diabetes. Int J Med Inform 2008;77:399-404.

14. Park MJ, Kim HS. Evaluation of mobile phone and Internet intervention on waist circumference and blood pressure in post-menopausal women with abdominal obesity. Int J Med Inform 2012;81:388-94.

15. Watson S, Woodside JV, Ware LJ, Hunter SJ, McGrath A, Cardwell CR, et al. Effect of a web-based behavior change program on weight loss and cardiovascular risk factors in overweight and obese adults at high risk of developing cardiovascular disease: randomized controlled trial. J Med Internet Res 2015; 17:e177.

16. Dâmaso AR. Saber Emagrecer. São Paulo: Weight Science; 2015.

17. Donnelly JE, Blair SN, Jakicic JM, Manore MM, Rankin JW, Smith BK, et al. American College of Sports Medicine Position Stand: appropriate physical activity intervention strategies for weight loss and prevention of weight regain for adults. Med Sci Sports Exerc 2009;41:459-71.

18. Biaggi RR, Vollman MW, Nies MA, Brener CE, Flakoll PJ, Levenhagen DK, et al. Comparison of air-displacement plethysmography with hydrostatic weighing and bioelectrical impedance analysis for the assessment of body composition in healthy adults. Am J Clin Nutr 1999;69:898-903.

19. Zimmet P, Alberti KG, Kaufman F, Tajima N, Silink M, Arslanian S, et al. The metabolic syndrome in children and adolescents: an IDF consensus report. Pediatr Diabetes 2007;8: 299-306.

20. Park MJ, Kim HS, Kim KS. Cellular phone and Internet-based individual intervention on blood pressure and obesity in obese patients with hypertension. Int J Med Inform 2009;78:704-10. 
21. Longin R, Grasse M, Aspalter R, Waldherr K. Effectiveness of the online weight reduction program KiloCoach ${ }^{\mathrm{TM}}$ and comparison with other evaluated commercial direct intervention and online programs. Obes Facts 2012;5:372-83.

22. Dunn C, Olabode-Dada O, Whetstone L, Thomas C, Aggarwal S, Nordby K, et al. Using synchronous distance education to deliver a weight loss intervention: a randomized trial. Obesity (Silver Spring) 2016;24:44-50.

23. Sousa P, Fonseca H, Gaspar P, Gaspar F. Usability of an internet-based platform (Next.Step) for adolescent weight management. J Pediatr (Rio J) 2015;91:68-74.

24. Krebs JD, Evans S, Cooney L, Mishra GD, Fruhbeck G, Finer $\mathrm{N}$, et al. Changes in risk factors for cardiovascular disease with body fat loss in obese women. Diabetes Obes Metab 2002;4: 379-87.

25. Corgosinho FC, Ackel-D’Elia C, Tufik S, Dâmaso AR, de Piano A, Sanches Pde L, et al. Beneficial effects of a multifaceted 1-year lifestyle intervention on metabolic abnormalities in obese adolescents with and without sleep-disordered breathing. Metab Syndr Relat Disord 2015;13:110-8.

26. Bianchini JA, da Silva DF, Hintze LJ, Antonini VD, Lopera CA, $\mathrm{McNeil} \mathrm{J}$, et al. Obese adolescents who gained/maintained or lost weight had similar body composition and cardiometabol- ic risk factors following a multidisciplinary intervention. J Exerc Sci Fit 2014;12:38-45.

27. Cotie LM, Prince SA, Elliott CG, Ziss MC, McDonnell LA, Mullen KA, et al. The effectiveness of eHealth interventions on physical activity and measures of obesity among workingage women: a systematic review and meta-analysis. Obes Rev 2018;19:1340-58.

28. Carnier J, de Piano A, de Lima Sanches P, Tock L, do Nascimento CM, Oyama LM, et al. The role of orexigenic and anorexigenic factors in an interdisciplinary weight loss therapy for obese adolescents with symptoms of eating disorders. Int J Clin Pract 2010;64:784-90.

29. Campos RM, Masquio DC, Corgosinho FC, Carvalho-Ferreira JP, Molin Netto BD, Clemente AP, et al. Relationship between adiponectin and leptin on osteocalcin in obese adolescents during weight loss therapy. Arch Endocrinol Metab 2018;62:275-84.

30. Rigamonti AE, Cicolini S, Caroli D, De Col A, Scacchi M, Cella SG, et al. Effects of a 3-week in-hospital body weight reduction program on cardiovascular risk factors, muscle performance, and fatigue: a retrospective study in a population of obese adults with or without metabolic syndrome. Nutrients 2020;12:1495. 